\title{
Dimensional Changes in Dental Plaster during the Setting Process
}

\author{
by \\ Minoru NISHIYAMA, Shigeru NISHIHARA and Masayoshi OHASHI
}

(Received 11 November 1985)

Key words: dental plaster, setting process, dimensional change, initial shrinkage, setting expansion, expansion initiation time, time constant, NIN apparatus.

\begin{abstract}
Summary
The authors, using an NIN apparatus, examined the dimensional changes occurring in dental plaster during the setting process from its slurry state immediately after being mixed to its solid state, and the following results were obtained: 1) Linear shrinkage that can be seen in the initial stage of the setting process shows a tendency to become larger when the water/powder ratio is increased, and the length of the specimen is shorter. 2) Expansion initiation time shows a tendency to be quickened when the water/powder ratio is reduced, but little difference due to the length of the specimen is recognizable. 3) The apparent linear expansion shows a tendency to become larger when the water/powder ratio is smaller, and when the length of the specimen is shorter. 4) Velocity (time constant) from the beginning of expansion to the maximum expansion is almost constant, being little influenced by the water/powder ratio and the length of the specimen.
\end{abstract}

\section{Introduction}

The authors have previously developed an NIN apparatus that makes it possible to measure the shrinkage and expansion of dental plaster during the setting process precisely and automatically ${ }^{[1]}$. In the present studies, the authors using this NIN apparatus, measured and examined the dimensional changes occurring in dental plaster during the setting process from its slurry state immediately after being mixed to its solid state.

\section{Materials and Methods}

1. Materials

Dental $\beta$-plaster* was used as the experimental material.

西山 實, 西原 滋, 大橋正敬: Department of Dental Materials, Nihon University School of Dentistry, 1-8-13 Kanda-Surugadai, Chiyoda-ku, Tokyo 101 Japan

*A2 type (Batch No. 07212), MARUISHI GYPSUM CO., Ltd., Tokyo, Japan. 

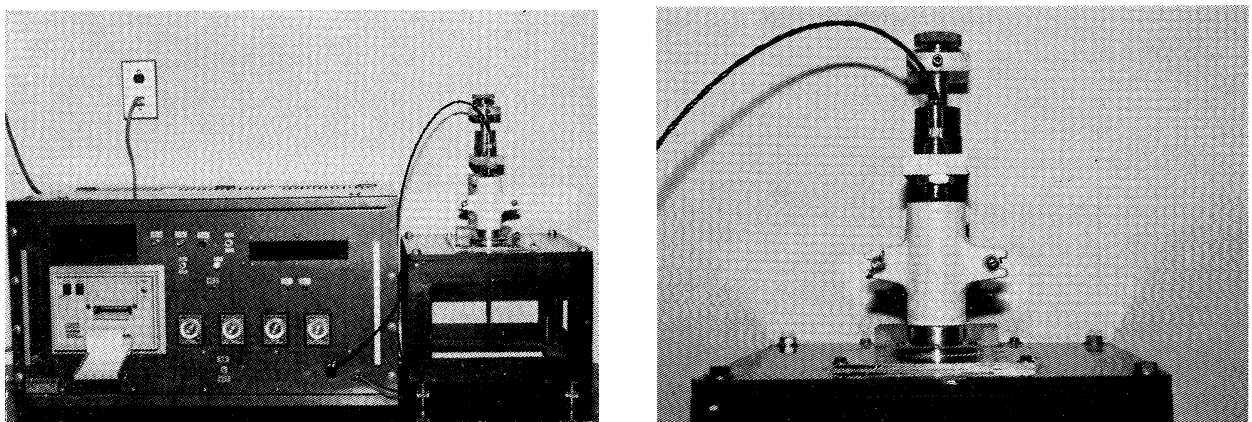

Fig. 1 The NIN apparatus used in the present study and an enlarged picture of its detector

\section{Methods}

1) Water/powder ratios

Water/powder ratios $(\mathrm{W} / \mathrm{P})$ were fixed at $0.45,0.55$ and 0.65 , with reference to the values used in dental laboratory work, $0.41-0.45^{[2]}$, and the manufacturer-recommended values, $0.63-0.67^{[3]}$. The temperature of the water used for mixing was $23 \pm 1^{\circ} \mathrm{C}$.

2) Length of specimen

Chosen lengths of specimens (L) were 20.0, 40.0 and $80.0 \mathrm{~mm}$.

3) Measuring method

Figure 1 shows the NIN apparatus ${ }^{[1]}$ used in the present experiment.

Fifty grams of plaster was added to a fixed amount of water in a mixing bowl, and after being left for 15 seconds, it was mixed with a hand-mixer attached to the bowl for 30 seconds at $120 \mathrm{rpm}$. The mixture was then shaken on a vibrator for 10 seconds to remove air bubbles. Next, the mixture was poured into the mold of the measuring apparatus, on which an aluminium plate $(0.50 \mathrm{~g}, \varnothing 8.5 \mathrm{~mm})$ was placed, and the sensor of the detector was placed in position, $2.500 \mathrm{~mm}$ above the aluminium plate. Measurement was carried out every 15 seconds for 60 minutes, beginning at 1 minute 30 seconds after the plaster powder had been added to the water. Measurement was repeated 5 times for every condition of the above-mentioned $\mathrm{W} / \mathrm{P}$ and $\mathrm{L}$.

Though the inner surface of the mold was very smooth, a layer of vaseline was applied to the inner surface to decrease the frictional resistance due to expansion.

The above-mentioned experiment was performed at a room temperature of $23 \pm 1{ }^{\circ} \mathrm{C}$ and $50 \pm 5 \%$ relative humidity.

\section{Results}

Figure 2 shows some examples of dimensional change curves for the setting process, and Table 1 shows the experimental results $(-\ell \max$ represents the maximum shrinkage, and $+\ell \max$ represents the maximum expansion). From these results, it is evident that plaster at first shows shrinkage immediately after being mixed, followed by an apparent expansion. However, the final measured values proved smaller than those taken at the start of measurement (zero point). 
Table 1 Results of the measurements

\begin{tabular}{|c|c|c|c|c|c|}
\hline Length (mm) & $\mathrm{W} / \mathrm{P}$ ratio & & $-\ell \max (\mathrm{mm})$ & $+\ell \max (\mathrm{mm})$ & $\begin{array}{c}\text { Expansion initiation } \\
\text { time (min) }\end{array}$ \\
\hline \multirow{21}{*}{$\mathrm{L}=20.0$} & \multirow{7}{*}{0.45} & 1 & -0.059 & -0.020 & 8.25 \\
\hline & & 2 & -0.076 & -0.044 & 7.25 \\
\hline & & 3 & -0.073 & -0.035 & 7.75 \\
\hline & & 4 & -0.062 & -0.023 & 7.50 \\
\hline & & 5 & -0.065 & -0.024 & 7.00 \\
\hline & & $\mathbf{M}$ & -0.067 & -0.029 & 7.55 \\
\hline & & SD & -0.007 & -0.010 & 0.417 \\
\hline & \multirow{7}{*}{0.55} & 1 & -0.080 & -0.054 & 9.75 \\
\hline & & 2 & -0.079 & -0.052 & 10.25 \\
\hline & & 3 & -0.076 & -0.046 & 10.25 \\
\hline & & 4 & -0.066 & -0.037 & 10.25 \\
\hline & & 5 & -0.074 & -0.042 & 9.75 \\
\hline & & $\mathrm{M}$ & -0.075 & -0.046 & 10.05 \\
\hline & & SD & 0.005 & 0.007 & 0.250 \\
\hline & \multirow{7}{*}{0.65} & 1 & -0.111 & -0.088 & 13.00 \\
\hline & & 2 & -0.082 & -0.055 & 12.25 \\
\hline & & 3 & -0.094 & -0.063 & 12.25 \\
\hline & & 4 & -0.090 & -0.066 & 12.25 \\
\hline & & 5 & -0.070 & -0.041 & 12.50 \\
\hline & & M & -0.089 & -0.062 & 12.45 \\
\hline & & SD & 0.015 & 0.017 & 0.283 \\
\hline \multirow{20}{*}{$\mathrm{L}=40.0$} & \multirow{7}{*}{0.45} & 1 & -0.106 & -0.039 & 7.00 \\
\hline & & 2 & -0.100 & -0.048 & 7.25 \\
\hline & & 3 & -0.121 & -0.060 & 8.25 \\
\hline & & 4 & -0.112 & -0.051 & 8.50 \\
\hline & & 5 & -0.101 & -0.037 & 7.00 \\
\hline & & $\mathbf{M}$ & -0.108 & -0.047 & 7.70 \\
\hline & & SD & 0.008 & 0.009 & 0.633 \\
\hline & \multirow{7}{*}{0.55} & 1 & -0.097 & -0.050 & 9.25 \\
\hline & & 2 & -0.116 & -0.063 & 10.50 \\
\hline & & 3 & -0.111 & -0.060 & 10.25 \\
\hline & & 4 & -0.133 & -0.082 & 9.00 \\
\hline & & 5 & -0.110 & -0.062 & 9.25 \\
\hline & & M & -0.113 & -0.063 & 9.70 \\
\hline & & SD & 0.013 & $\begin{array}{r}0.011 \\
-0.052\end{array}$ & $\frac{0.600}{1125}$ \\
\hline & \multirow{6}{*}{0.65} & 1 & $\begin{array}{l}-0.099 \\
-0.102\end{array}$ & $\begin{array}{l}-0.052 \\
-0.053\end{array}$ & $\begin{array}{l}11.25 \\
12.50\end{array}$ \\
\hline & & $\begin{array}{l}2 \\
3\end{array}$ & $\begin{array}{l}-0.102 \\
-0.123\end{array}$ & $\begin{array}{l}-0.053 \\
-0.080\end{array}$ & $\begin{array}{l}12.50 \\
13.00\end{array}$ \\
\hline & & 4 & -0.125 & -0.078 & 12.75 \\
\hline & & 5 & -0.134 & -0.090 & 12.25 \\
\hline & & $\mathbf{M}$ & -0.116 & -0.070 & 12.75 \\
\hline & & SD & 0.015 & 0.017 & 0.600 \\
\hline \multirow{21}{*}{$L=80.0$} & \multirow{7}{*}{0.45} & 1 & -0.129 & -0.047 & 9.00 \\
\hline & & 2 & -0.140 & -0.055 & 8.25 \\
\hline & & 3 & -0.150 & -0.062 & 7.75 \\
\hline & & 4 & -0.128 & -0.046 & 8.00 \\
\hline & & 5 & -0.131 & -0.050 & 8.25 \\
\hline & & $M$ & -0.135 & -0.052 & 8.25 \\
\hline & & SD & 0.009 & 0.006 & 0.417 \\
\hline & \multirow{7}{*}{0.55} & 1 & -0.190 & -0.118 & 10.25 \\
\hline & & 2 & -0.183 & -0.111 & 10.50 \\
\hline & & 3 & -0.158 & -0.083 & 10.00 \\
\hline & & 4 & -0.168 & -0.098 & 10.00 \\
\hline & & 5 & -0.161 & -0.094 & 9.25 \\
\hline & & M & -0.172 & -0.100 & 10.00 \\
\hline & & SD & 0.013 & 0.013 & 0.417 \\
\hline & \multirow{7}{*}{0.65} & 1 & -0.186 & -0.118 & 12.75 \\
\hline & & 2 & -0.190 & -0.123 & 12.00 \\
\hline & & 3 & -0.189 & -0.130 & 13.75 \\
\hline & & 4 & -0.162 & -0.099 & 13.00 \\
\hline & & 5 & -0.178 & -0.116 & 12.25 \\
\hline & & $M$ & -0.181 & -0.117 & 12.75 \\
\hline & & SD & 0.011 & 0.011 & 0.583 \\
\hline
\end{tabular}




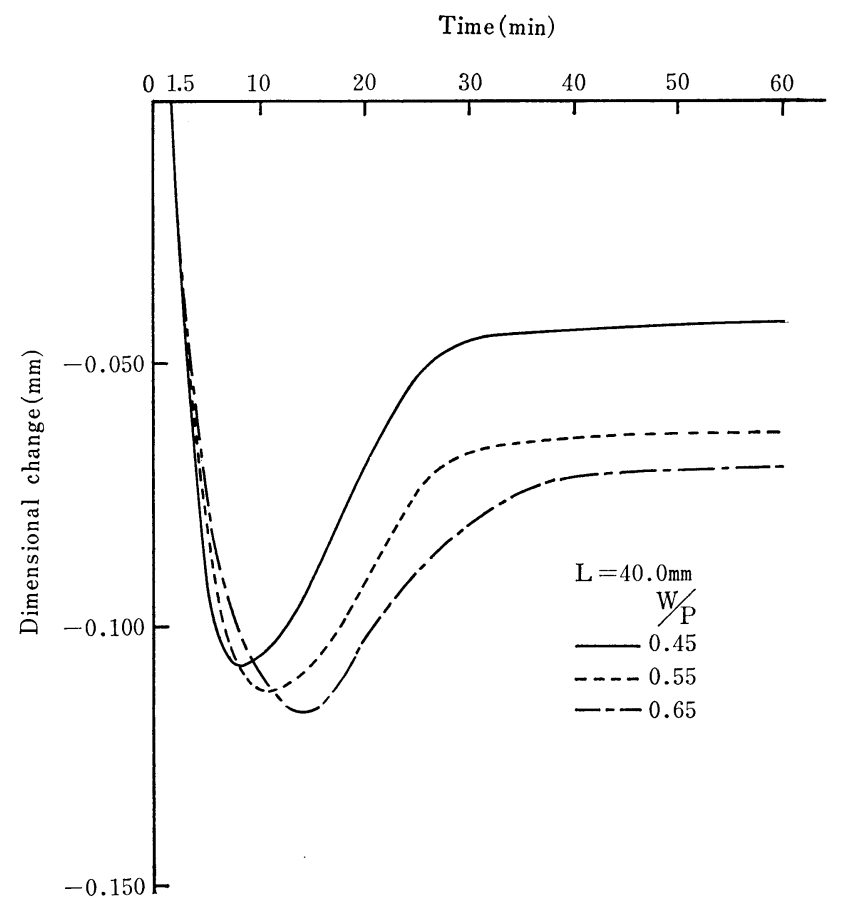

Fig. 2 Some examples of dimensional change curves for the setting process

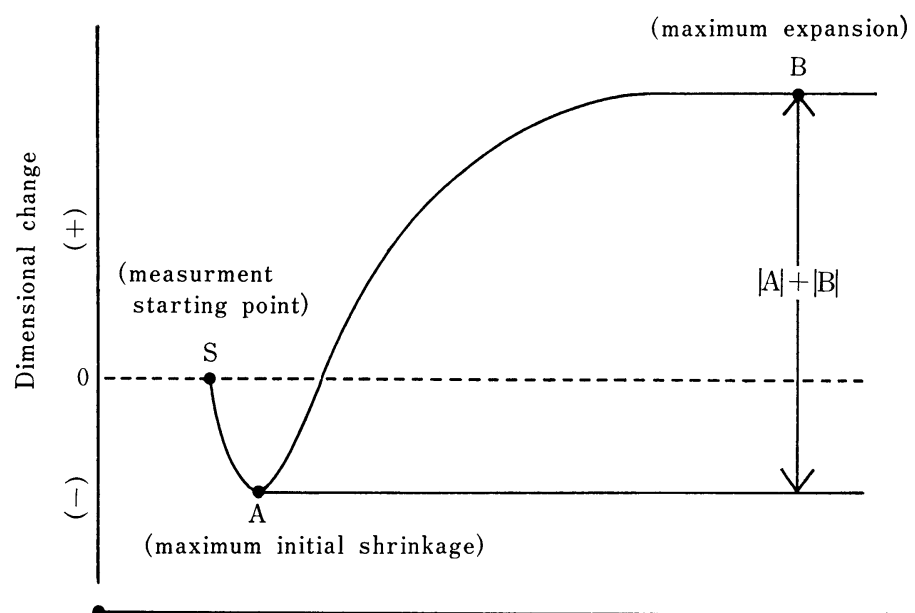

$\mathrm{P}$ (point $\mathrm{P}$ indicates that plaster powder was added to water)

Time $\rightarrow$

Fig. 3 Curve of shrinkage and expansion during the setting process 
In general, the shrinkage and expansion during the setting process can be represented by the curve shown in Fig. 3. That is, from the zero point, a measurement starting point set at random, the curve at first shows shrinkage and then expansion before reaching a plateau. A in Fig. 3 represents the maximum shrinkage, that is, the expansion initiation point, and $\mathrm{B}$ the maximum expansion. Again, the expansion can be represented by $|\mathrm{A}|+|\mathrm{B}|$, with the maximum shrinkage value regarded as the original point.

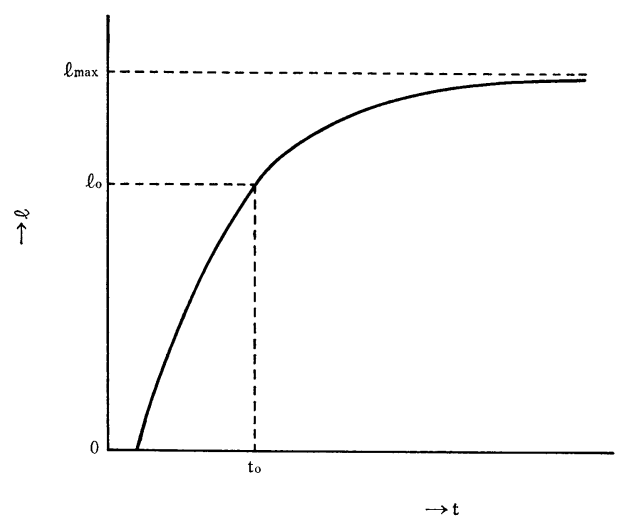

Fig. 4 Expansion curve

On the other hand, Fig. 4 shows the curve of expansion taken out of Fig. 3. This type of curve can be presumed to be a function of time $t$ as shown below:

$$
\begin{aligned}
& \ell o=\ell \max \left(1-\mathrm{e}^{\frac{\text { to }}{\mathrm{T}}}\right), \quad(\mathrm{e}=2.71828 \ldots \mathrm{T}: \text { time constant }) \\
& \ell o-\ell \max =-\ell \max \cdot \mathrm{e}^{\frac{\text { to }}{\mathrm{T}}} \\
& \left(1-\frac{\ell o}{\ell \max }\right)=\mathrm{e}^{\frac{\mathrm{to}}{\mathrm{T}}}
\end{aligned}
$$

taking logarithms of both sides:

$$
\log _{10} \mathrm{e}\left(1-\frac{\ell o}{\ell \max }\right)=\frac{\text { to }}{\mathrm{T}} \log _{10} \mathrm{e}
$$

left side as $y$-axis:

$$
y=\frac{\log _{10} \mathrm{e}}{\mathrm{T}} \text { to }
$$

and:

$$
\mathrm{A}=\frac{\log _{10} \mathrm{e}}{\mathrm{T}}
$$

then, formula (3) can be a linear function of $t$, as shown below:

$$
y=\mathrm{A} \cdot \mathrm{to}
$$


Then, the measured values, substituted into formula (4), plotted on the graph, prove to be almost linear, and thus it is confirmed that the setting expansion curve can be represented by formula (1).

Consequently, from the expansion curve shown in Fig. 4, regarding the maximum shrinkage as the original point, the maximum expansion as $\Delta \ell \max$ and the time constant as $\mathrm{T}, \Delta \ell o$, the expansion at any point in time can be expressed as follows:

$$
\Delta \ell o=\Delta \ell \max \left(1-\mathrm{e}^{\frac{\mathrm{to}}{\mathrm{T}}}\right)
$$

By determining $\mathrm{T}$ from this formula, the relative velocity of expansion of the plaster during the setting process, from the beginning to the finish, can be obtained.

Based on the facts mentioned above, and the results shown in Table 1, linear shrinkage calculated from the maximum shrinkage, expansion initiation time, apparent linear expansion, determined with the maximum shrinkage as the original point, and the time constant were obtained.

\section{Linear shrinkage}

Figure 5 shows the relationship between linear shrinkage and $\mathrm{W} / \mathrm{P}$ and $\mathrm{L}$.

Linear shrinkage shows a tendency to increase when $\mathrm{W} / \mathrm{P}$ is larger, which in turn occurs when $\mathrm{L}$ is smaller.

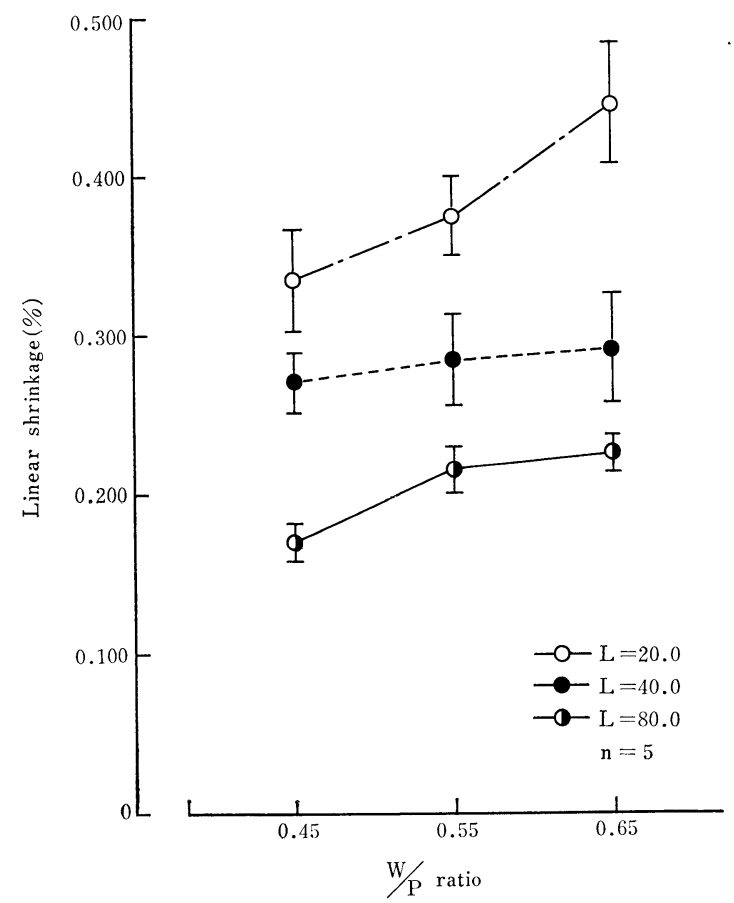

Fig. 5 Relationship between linear shrinkage and W/P and L 


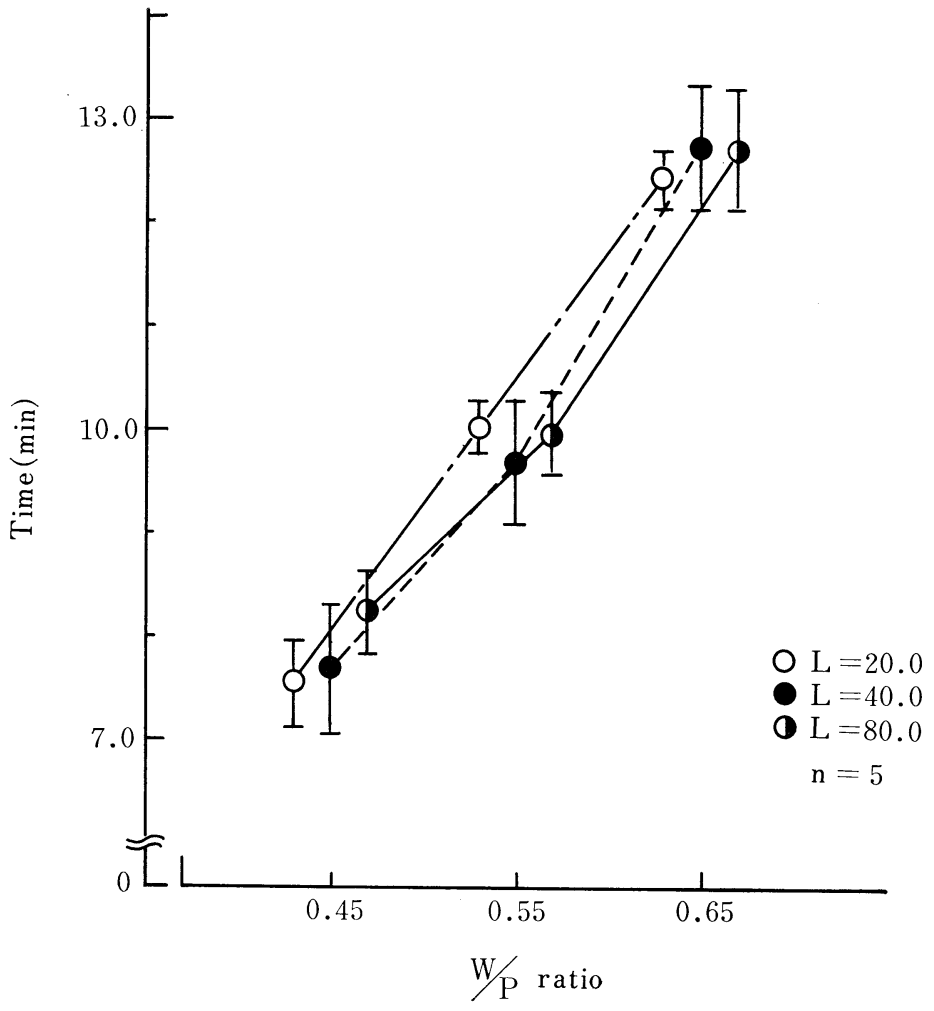

Fig. 6 Relationship between expansion initiation time and W/P and L

\section{Expansion initiation time}

Figure 6 shows the relationship between expansion initiation time and W/P and L.

Expansion initiation time shows a tendency to be earlier when $\mathrm{W} / \mathrm{P}$ is smaller, but hardly any difference due to $\mathrm{L}$ can be recognized.

3. Apparent linear expansion and L.

Figure 7 shows the relationship between apparent linear expansion and W/P

Apparent linear expansion shows a tendency to increase when both W/P and $\mathrm{L}$ are smaller.

4. Time constant

Figure 8 shows the relationship between the time cons tant and W/P and L.

As can be seen, $\mathrm{W} / \mathrm{P}$ and $\mathrm{L}$ have apparently no influence on the time constant. 


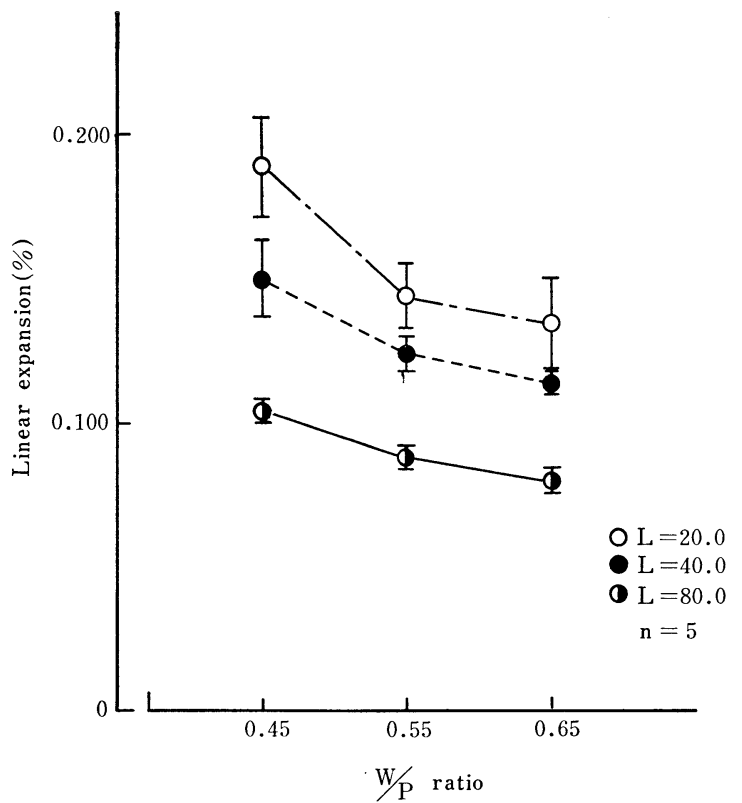

Fig. 7 Relationship between apparent linear expansion and W/P and L

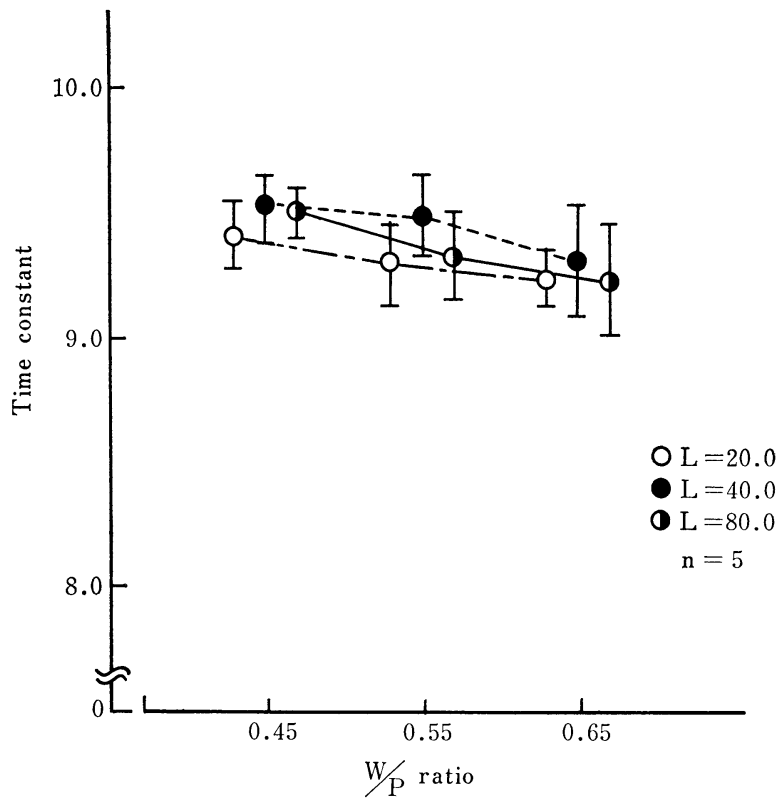

Fig. 8 Relationship between time constant and W/P and L 


\section{Discussion}

\section{Initial shrinkage}

The experimental results of the present study prove that shrinkage can be recognized in the initial stage of the setting process. This shrinkage phenomenon can hardly be considered to be due to flow of the plaster mixture, as the measurement load was only $0.50 \mathrm{~g}$ (aluminium plate), a negligible weight, as stated before. Therefore, it is appropriate to consider that the phenomenon is real shrinkage due to the formation of plaster dihydrate, as expressed in the theory of plaster setting ${ }^{[4]}$. Reports have already been published with regard to the initial shrinkage phenomenon $^{[4-6]}$, but in the present studies, the change appearing in the initial stage can be discerned more clearly, compared with that in other reports, as the measurement point (zero point) was set 1.5 minutes after the plaster was added to the water.

With regard to the relationship between linear shrinkage and W/P (Fig. 5), it was proved that linear shrinkage becomes larger with increased $\mathrm{W} / \mathrm{P}$. This coincides with a report ${ }^{[7]}$ that the larger $\mathrm{W} / \mathrm{P}$ becomes, the larger the initial shrinkage, which can theoretically be considered due to the evaporation of excess water ${ }^{[7]}$ unnecessary for the reaction with plaster powder.

On the other hand, with regard to the relationship between linear shrinkage and L (Fig. 5), it was shown that the larger the value of L, the smaller the linear shrinkage becomes. From the standpoint of the assumption that the initial shrinkage of plaster is due to the evaporation of excess water, it can be presumed that the greater the value of $\mathrm{L}$, the less the evaporation of water becomes, the exposed area becoming smaller as a whole. Consequently, the initial shrinkage is suppressed when $\mathrm{L}$ is greater.

\section{Setting expansion}

Theoretically, the volume shrinkage of plaster in the setting process is approximately $7.1 \%$. However, it does show expansion. This is presumably due to the fact that in the setting mechanism, hemihydrate, reacting with water, is turned into dihydrate, the crystals of the plaster becoming needle-like ${ }^{[5]}$. In the present experiments, however, the final result showed shrinkage as a whole, with values smaller than those of the measurement initiation point (zero point) (Table 1).

This is presumably due to the period at which the measurement initiation point was set. That is, the initial shrinkage, and therefore, in turn, the expansion, differs according to whether the zero point is set at an earlier stage after mixing or some time later. For instance, in the present studies, the zero point was set at 1.5 minutes after the plaster was added to the water, while if it had been set after 6 minutes $^{[8,9]}$ or after 8 minutes $^{[6]}$, all the final values shown in Fig. 2 would have shown expansion. Consequently, when examining the setting expansion properties of plaster, the measurement initiation point should not be set at random but rather at the point of maximum shrinkage (expansion initiation point).

\section{1) Expansion initiation time}

Expansion initiation time was more delayed when W/P was larger (Fig. 6). This coincides with a report ${ }^{[7]}$ which states that the larger $\mathrm{W} / \mathrm{P}$ becomes the longer the shrinkage period. The presumption is that the density of the plaster particles is so 
small that the distance between crystals becomes larger, and that particle interaction along with crystal growth is more delayed.

On the other hand, from the fact that there was little difference due to $\mathrm{L}$, it appears that expansion initiation time is almost constant, whatever the size of the specimen might be, $\mathrm{L}$ having little influence.

2) Apparent linear expansion

Apparent linear expansion became larger when W/P was smaller (Fig. 7). This coincides with the theory that the setting expansion becomes larger when $\mathrm{W} / \mathrm{P}$ is smaller ${ }^{[5]}$. That is, when $\mathrm{W} / \mathrm{P}$ is large, the number of nuclei of crystallization per unit volume is small, and consequently the distance between nuclei becomes large, so that crystals of dihydrate can develop freely without interacting with one another, and consequently, growth toward the outside does not occur. The opposite phenomenon may occur when $\mathrm{W} / \mathrm{P}$ is small.

With regard to the influence of $\mathrm{L}$, it was proved that the smaller the value of $\mathrm{L}$, the larger the apparent linear expansion became (Fig. 7). The presumption is that if the specimen is short, the frictional resistance of the mold is small, so that the expansion strength becomes large. This tendency coincides with the report that the setting expansion increases when the length of the specimen is shorter ${ }^{[10]}$.

3) Expansion velocity (time constant)

As described above, the velocity of setting expansion until a maximum is attained, can be relatively compared to the time constant. From the experimental results (Fig. 8), it was apparent that the time constant is little influenced by differences of $\mathrm{W} / \mathrm{P}$ and $\mathrm{L}$. That is, up to the expansion initiation point, as described above, a difference can be seen according to the influence of $\mathrm{W} / \mathrm{P}$, but from the beginning of expansion to the maximum, hardly any difference of velocity (time constant) is recognizable in any case. From this fact it is presumed that the difference in the length of setting time caused by $\mathrm{W} / \mathrm{P}$ is due to differences in time up to the beginning of setting expansion.

\section{Conclusions}

The authors, using an NIN apparatus and commercially available $\beta$-plaster as the experimental material, examined the dimensional changes occurring in plaster during the setting process from its slurry state immediately after being mixed to the final solid state, using various water/powder ratios and lengths of specimen. The results were as follows:

1) Linear shrinkage in the initial stage of the setting process showed a tendency to increase when $\mathrm{W} / \mathrm{P}$ was larger and the length of specimen shorter.

2) Expansion initiation time showed a tendency to be earlier when $W / P$ was smaller, but there was little difference due to the length of the specimen.

3) Apparent linear expansion showed a tendency to increase when $\mathrm{W} / \mathrm{P}$ was smaller and the length of the specimen shorter.

4) Velocity (time constant) from the beginning of expansion to the maximum expansion was almost constant, and not influenced by $\mathrm{W} / \mathrm{P}$ or the length of the specimen. 


\section{References}

[1] Nishiyama, M. et al.: Development of measurement apparatus for expansion and shrinkage during setting, using a non-contact micro displacement meter, J. Nihon Univ. Sch. Dent., 23, 159-169, 1982

[2] Katayama, K.: A study on the testing consistency of dental gypsum products, JDMA, 38, 179-195, 1981 (in Japanese)

[3] Horibe, T. and Окамото, K.: Modern model materials and investments, J. Dent. Med., 14, 369-381, 1981 (in Japanese)

[4] Aoyagi, Y.: Studies on setting expansion of dental stone (Part 1), JDMA., 10, 187-199, 1969 (in Japanese)

[5] Phillips, R.W.: Science of dental materials, 7th ed., 64-67, Saunders, Philadelphia, U.S.A., 1973

[6] Kato, M. et al.: The dimensional change of dental stone inside impression material (Part 1), Aichi Gakuin J Dent Science, 14, 386-390, 1977 (in Japanese)

[7] OTANi, H. and OKADA, N.: Weight reduction and porosity formation of hydrated dental stone due to excess water evaporation, JDMA, 15, 195-199, 1974 (in Japanese)

[8] JIS T 6604, 1976

[9] JIS T 6605, 1976

[10] Yamane, S. and Wakamatsu, Y.: Studies on accuracy of cast products (Part 3), JDMA, 8, 60-64, 1967 (in Japanese) 